\title{
Preparation, characterization, and post-synthetic modification of layered MCM-22 zeolite precursor
}

\author{
RIDDHI THAKKAR and RAJIB BANDYOPADHYAY* \\ School of Technology, Department of Science, Pandit Deendayal Petroleum University, Gandhinagar, \\ Gujarat 382 007, India \\ E-mail: rajib.bandyopadhyay@ sot.pdpu.ac.in
}

MS received 7 April 2017; revised 18 July 2017; accepted 31 July 2017; published online 19 September 2017

\begin{abstract}
Hydrothermal synthesis of MCM-22(P) was carried out with two different silica sources, colloidal silica $(28 \%)$ and silicic acid with different gel composition. The synthesis was carried out in stirring and static conditions with different crystallization time. MCM-22(P) modified with swelling-sonication method resulted in swollen MCM-22, while alkali treatment yielded desilicated MCM-22. The materials were characterized by X-ray diffraction, low-angle XRD, FE-SEM-EDX, FT-IR, TGA, $\mathrm{N}_{2}$ adsorption and $\mathrm{NH}_{3}-\mathrm{TPD}$ analysis. The results revealed that MCM-22 has a layered sphere, doughnut like morphology and after modification, swollen and broken sphere was observed. Physicochemical analysis revealed that the materials' mesoporosity increased and acidity also changed. Energy dispersive X-ray analysis revealed the high amount of desilication in alkali-treated MCM-22(P).
\end{abstract}

Keywords. Colloidal silica; silicic acid; MCM-22(P); swelling-sonication; alkali-treatment.

\section{Introduction}

Zeolites are microporous, inorganic tetrahedrally coordinated polyaluminosilicate and a promising material having superior catalytic properties. In heterogeneous catalysis, layered zeolites are catalytic materials that combine excellent activity in acid-catalysed reactions with a high stability. MCM-22 is one of such layered zeolites having MWW topology ${ }^{1}$ (MCM-t $\underline{\text { Wenty-t }}$ Wo, Mobil Composition of Matter with sequence number twenty-two) with layered pockets of 10 member ring and surface pockets of 12 member ring channels ${ }^{2}$ (Figure 1). Layered as-made MCM-22 has 2D structure while calcined MCM-22 has 3D structure. This is observed due to the external surface of each zeolitic sheet having elevated amount of silanol groups ( $\mathrm{Si}-$ $\mathrm{OH})$. After the calcination process, these react with other silanols present in contiguous layer covalently condensing between them and facilitating the formation of 3D MWW zeolitic structure. ${ }^{3-5}$

MCM-22 is first layered zeolite which is hydrothermally synthesized in static and stirring conditions. ${ }^{6}$
Modifications of MCM-22(P) is possible by methods like alkaline treatment and swelling-sonication which may change its morphology and textural properties. ${ }^{7}$ The as-made zeolites are called as a precursor which can be used for further modifications.

The objective of this work is to study the synthesis of parent MCM-22, its modification and comparison of physicochemical properties between parent and modified MCM-22.

\section{Experimental}

\subsection{Materials}

Layered zeolite (MCM-22) was synthesized hydrothermally with gel composition $2.7 \mathrm{Na}_{2} \mathrm{O}: \mathrm{Al}_{2} \mathrm{O}_{3}: 30 \mathrm{SiO}_{2}: 1347 \mathrm{H}_{2} \mathrm{O}$ : 15HMI. The synthesis was performed using two different silica sources, colloidal silica (28\%) in stirring condition and silicic acid (Loba-Chemie) in a static condition, respectively. Sodium aluminate $\left(\mathrm{Al}_{2} \mathrm{O}_{3}-53.2 \%, \mathrm{Na}_{2} \mathrm{O}-43.9 \%\right.$ SigmaAldrich) was used as alumina source. Hexamethyleneimine

\footnotetext{
*For correspondence

Electronic supplementary material: The online version of this article (https://doi.org/10.1007/s12039-017-1366-3) contains supplementary material, which is available to authorized users.
} 

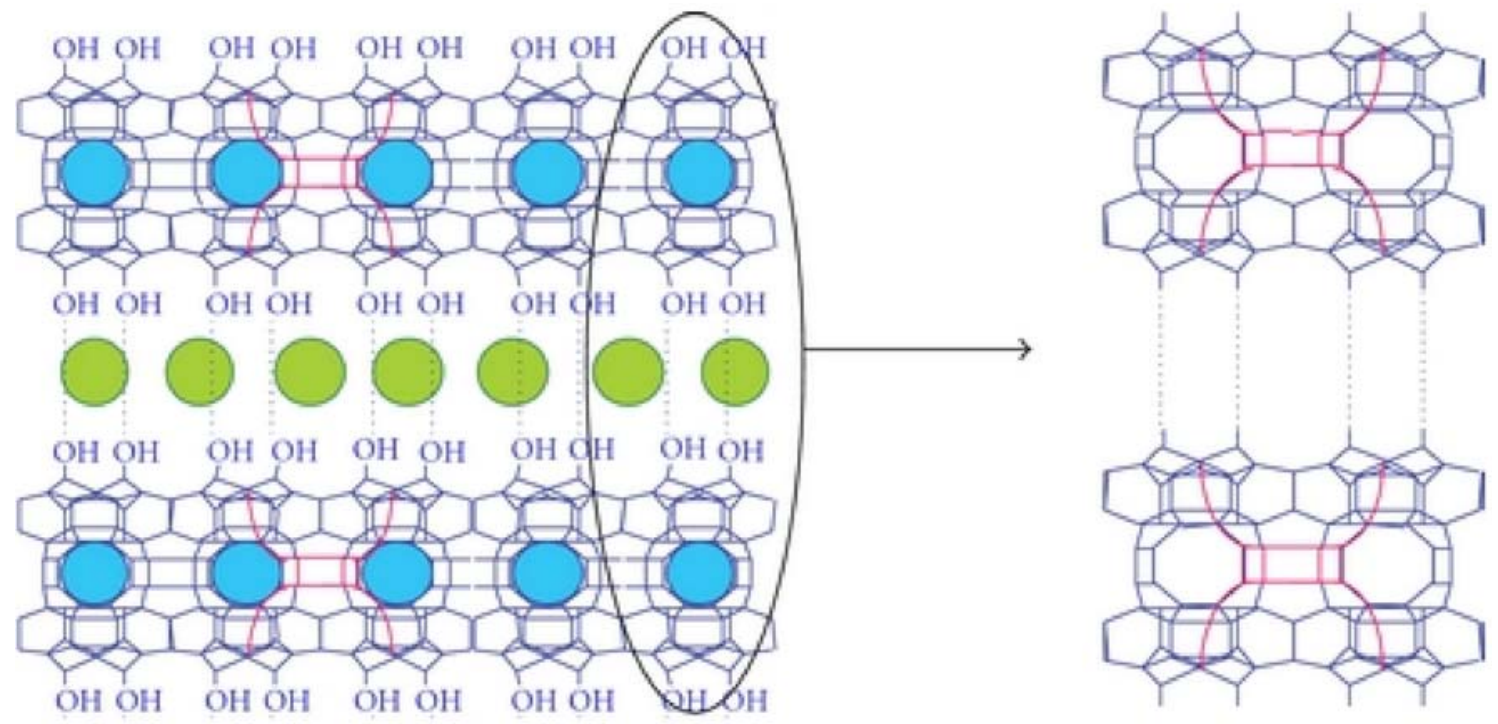

$12 \mathrm{MR}$ semicups

$10 \mathrm{MR}$ sinusoidal channels

Organic template: hexamethylenimine

Si-OH groups condensation

Figure 1. MWW topology of layered MCM-22 precursor. $^{8,9}$

(99\% Sigma-Aldrich) worked as an organic structure directing agent. The sodium hydroxide was used as alkali source and balance the sodium content and $\mathrm{pH}$ of the synthesis gel. The solvent for the gel was water. Modifications on MCM-22(P) was performed using a surface active agent, tetrapropylammonium hydroxide (TPAOH 40\%) and swelling agent, hexadecyltrimethylammonium chloride (CTAB; Sigma-Aldrich) for the swelling-sonication method. The modification by alkalitreatment was performed using excess sodium hydroxide and TPAOH.

2.1a Synthesis of $M C M-22(P)$ : Initially, a series of experiments were performed to optimize synthetic parameters of the layered zeolite MCM-22. It was synthesized following published procedure. ${ }^{10,11}$ Hydrothermal synthesis was carried out with two different silica sources, colloidal silica and silicic acid with a gel composition: $2.7 \mathrm{Na}_{2} \mathrm{O}: \mathrm{Al}_{2} \mathrm{O}_{3}: 30 \mathrm{SiO}_{2}$ : $1347 \mathrm{H}_{2} \mathrm{O}: 15 \mathrm{HMI}$. The gel was transferred to stirring and static autoclaves for hydrothermal synthesis at $423 \mathrm{~K}$ for $110 \mathrm{~h}$ and $240 \mathrm{~h}$, respectively. The as-synthesized MCM-22 was filtered, washed with deionized water and dried at $393 \mathrm{~K}$ for $12 \mathrm{~h}$. The dried zeolite was further crushed to fine powder and calcined at $823 \mathrm{~K}$ for $10 \mathrm{~h}$ in a flow of dry air to remove organic template. The H-form of MCM-22 was obtained by repeated ammonium exchange with ammonium nitrate solution $(10 \%)$ followed by calcination at $823 \mathrm{~K}$ overnight.

\subsection{Preparation of modified MCM-22(P)}

2.2a Swelling-sonication method: MCM-22(P) slurry was mixed with hexadecyltrimethylammonium bromide solution (29 wt.\%) and tetrapropylammonium hydroxide (40 wt. $\%$ ), and refluxed for $16 \mathrm{~h}$ at $80^{\circ} \mathrm{C}$ to create swelling. ${ }^{12-15}$ The slurry was placed in an ultrasound bath $(50 \mathrm{~W}, 20 \mathrm{kHz})$ for $1 \mathrm{~h}$. Finally, pH of the slurry was maintained below 2 by adding concentrated $\mathrm{HCl}$, yielding modified MCM-22(P). The modified slurry was filtered, washed and dried overnight at $373 \mathrm{~K}$, and calcined at $823 \mathrm{~K}$ for $10 \mathrm{~h}$.

2.2b Alkali-treatment method: The alkaline treatment of MCM-22(P) was carried out in the presence of a mixture of $0.1 \mathrm{M} \mathrm{NaOH}$ and $40 \%$ Tetrapropylammonium hydroxide at $70^{\circ} \mathrm{C}$ for $5 \mathrm{~h}$, yielding alkali-treated MCM-22(P). ${ }^{16-18}$ The other procedure of filtering, drying, calcination and converting to $\mathrm{H}$-form of catalyst remained same as that of MCM-22.

\subsection{Physicochemical characterization}

Crystallinity and phase purity was determined by powder Xray diffraction for both as-made and calcined samples using PANalytical X'Pert pro XRD equipment. The operational parameter was $\mathrm{CuK} \alpha$ radiation at $40 \mathrm{kV} / 40 \mathrm{~mA}$, with a goniometer speed of $2^{\circ} / \mathrm{min}$ and a step of $0.02^{\circ}$ in the $2 \theta$ 
range from $5^{\circ}$ to $50^{\circ}$. The morphology and elemental composition of samples were evaluated using field emission scanning electron microscopy combined with energy dispersive X-ray ZEISS equipment. FT-IR was performed on PerkinElmer's FTIR spectrometer model 'Spectrum Two'. TGA was performed on Mettler Toledo Star ${ }^{\mathrm{e}}$ system. Zeolite's textural properties were confirmed using Quantachrome Autosorb IQ nitrogen adsorption-desorption pore size and surface area analyzer. The acidity of catalyst was carried out by $\mathrm{NH}_{3}-\mathrm{TPD}$ method using Micromeritics Autochem 2920.

\section{Results and Discussion}

\subsection{Influence of the silica source/synthesis procedures}

The effectiveness of the silica sources and synthesis conditions during the synthesis of MCM-22 was observed in the difference in crystallinity. MCM-22 synthesized using silicic acid in static condition shows high crystallinity and purity, while that synthesized using colloidal silica in stirring condition has comparatively low crystallinity. The powder XRD pattern of as-made and calcined MCM-22 are shown in the Figures S1 and S2 of Supplementary Information (SI).

MCM-22 synthesized from two different silica sources having different physical properties influences the shape of the layered structure of MCM-22, and this was observed in FE-SEM images. MCM-22 (silicic acid) shows doughnut like morphology having paper-like layers, and MCM-22 (colloidal silica) shows spherical shape having a regular arrangement of layers. However, MCM-22(P) formed from colloidal silica was further modified with swelling-sonication and alkaline treatment, showing significant changes in morphology. Modification shows swelling of parent sphere and breaking of a sphere with agglomerated layers. FE-SEM images are shown in the Supplementary Information (SI), Figure S3.

Zeolite framework properties like framework stretching and bending vibrations are studied through FT-IR analysis. The bending and stretching observed in midinfrared due to internal vibrations of the $\mathrm{TO}_{2}$ tetrahedra $(\mathrm{T}=\mathrm{Si}$ or $\mathrm{Al})$. Zeolite's framework vibration of calcined samples was observed in the range of $1300-400 \mathrm{~cm}^{-1}$. The analysis results are shown in the Figure S4 of Supplementary Information (SI).

\subsection{Analysis of synthesized MCM-22 and modified MCM-22(P)}

MCM-22 prepared from two different silica sources has high crystallinity than modified MCM-22(P). Low angle peak below $2 \theta=2^{\circ}$ was observed in the case of modified MCM-22 (swelling-sonicated) indicating the formation of mesoporosity. XRD pattern shows that the alkali-treated sample maintains the crystal structure of the MCM-22 zeolite. Generally, the alkali-treatment process under certain conditions can extract $\mathrm{Si}$ atoms from the framework and partially damage the topology of the zeolite showing decreased crystallinity than their parent zeolite. The XRD pattern in Figure 2 shows a mixture of different peaks comprising sharp, broad and tiny peaks.

Thermal stability of zeolites is one of the important features that make zeolites applicable as selective sorbents and potential catalysts. Kinetics of the dehydration of zeolites is normally studied between $25^{\circ} \mathrm{C}$ to $250^{\circ} \mathrm{C}$.
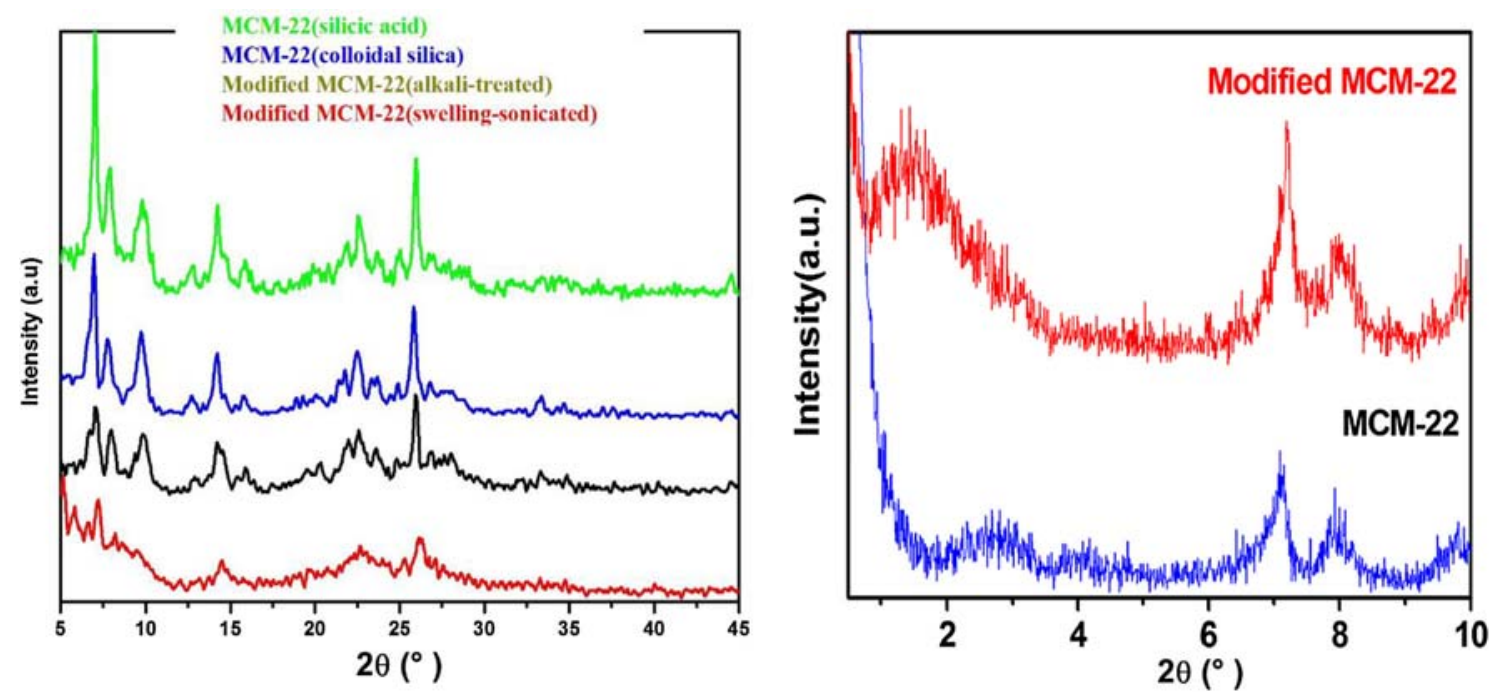

Figure 2. Powder XRD and LAXRD of parent and modified MCM-22. 
Table 1. Chemical composition and textural properties of all zeolite samples studied.

\begin{tabular}{lcccccc}
\hline Name & $\mathrm{SiO}_{2} / \mathrm{Al}_{2} \mathrm{O}_{3}$ ratio & $\mathrm{S}_{\mathrm{BET}}\left(\mathrm{m}^{2} \mathrm{~g}^{-1}\right)^{\mathrm{a}}$ & $\mathrm{S}_{\text {ext }}\left(\mathrm{m}^{2} \mathrm{~g}^{-1}\right)^{\mathrm{b}}$ & $\mathrm{V}_{\text {tot }}\left(\mathrm{cm}^{3} \mathrm{~g}^{-1}\right)^{\mathrm{c}}$ & $\mathrm{V}_{\text {mic }}\left(\mathrm{cm}^{3} \mathrm{~g}^{-1}\right)^{\mathrm{c}}$ & $\mathrm{V}_{\text {mas }}\left(\mathrm{cm}^{3} \mathrm{~g}^{-1}\right)^{\mathrm{c}}$ \\
\hline $\begin{array}{l}\text { MCM-22 } \\
\text { MCM-22 }\end{array}$ & 30.30 & 432.7 & 94.63 & 0.2232 & 0.1442 & 0.0790 \\
$\begin{array}{l}\text { Modified } \\
\text { MCM-22 }\end{array}$ & 30.30 & 377.4 & 123.4 & 0.2606 & 0.1121 & 0.0285 \\
$\begin{array}{l}\text { Modified } \\
\text { MCM-22 }\end{array}$ & 14.36 & 463.4 & 427.6 & 0.5627 & 0.0980 & 0.4647 \\
\hline
\end{tabular}

${ }^{\mathrm{a}} \mathrm{S}_{\mathrm{BET}}=$ BET surface area. ${ }^{\mathrm{b}} \mathrm{S}_{\mathrm{ext}}=$ external surface area. ${ }^{\mathrm{c}} \mathrm{V}_{\text {tot }}, \mathrm{V}_{\text {mic }}$ and $\mathrm{V}_{\text {mas }}$ correspond to the total, micro and meso pore volume of the zeolites. ${ }^{\mathrm{d}} \mathrm{MCM}-22=$ synthesized by silicic acid. ${ }^{\mathrm{e}} \mathrm{MCM}-22=$ synthesized by colloidal silica. ${ }^{\mathrm{f}}$ MCM-22 = swelling-sonicated. ${ }^{\mathrm{g}} \mathrm{MCM}-22=$ alkali-treated. The silica/alumina ratio from EDX analysis.
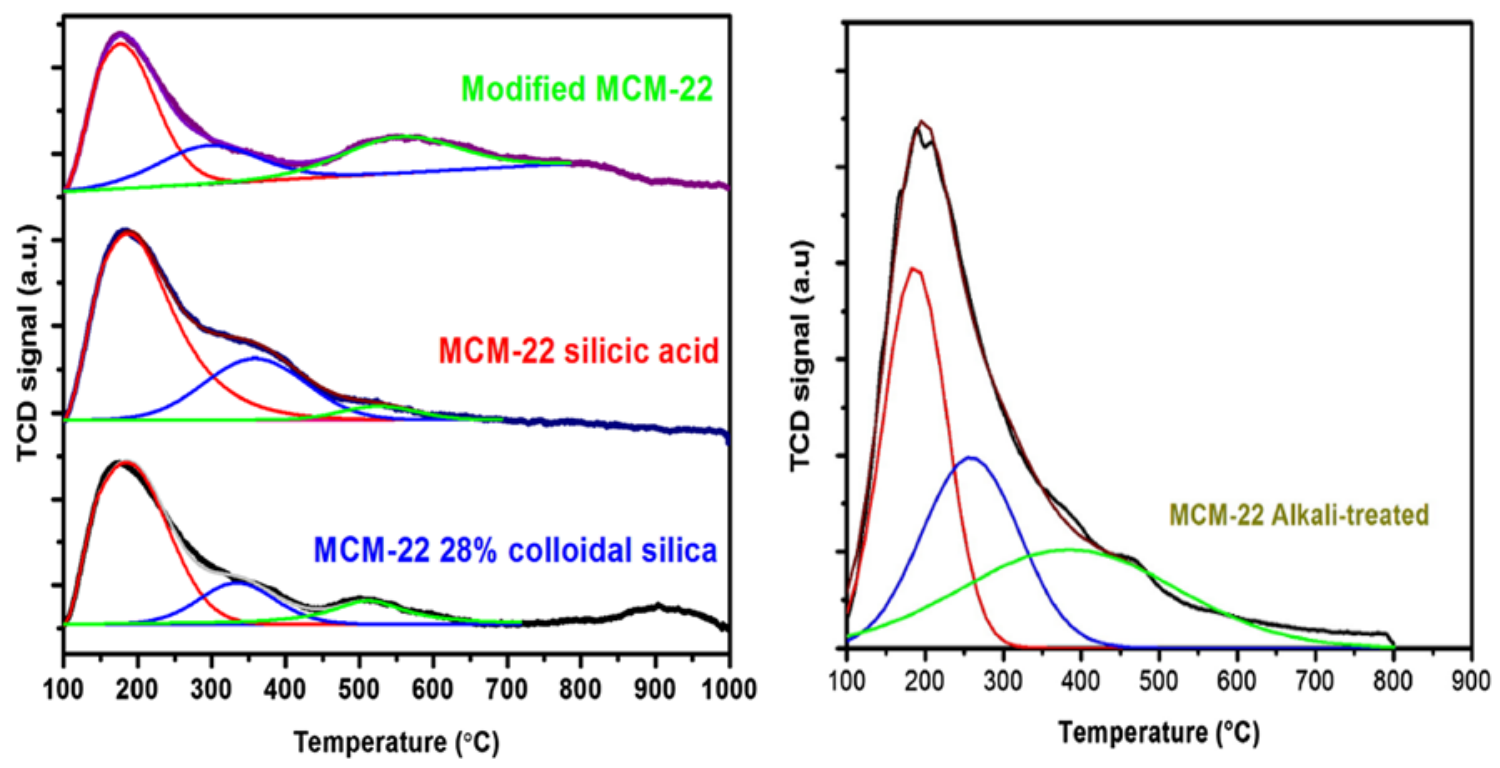

Figure 3. Deconvoluted ammonia TPD of zeolite samples.

Table 2. NH3-TPD qualitative and quantitative results.

\begin{tabular}{|c|c|c|}
\hline Material name & Temperature $\left({ }^{\circ} \mathrm{C}\right)$ & Acid sites concentration $(\mathrm{mmol} \mathrm{NH} / \mathrm{g})$ \\
\hline Modified MCM-22a & 178.7 & 0.281 \\
\hline Modified MCM-22a & 546.9 & 0.077 \\
\hline MCM-22 (silicic acid) & 183.2 & 0.526 \\
\hline MCM-22 (colloidal silica) & 176.5 & 0.423 \\
\hline
\end{tabular}

${ }^{\mathrm{a}} \mathrm{MCM}-22$ = swelling-sonicated modification.

Oxidative decomposition of the occluded organic template is observed at $250^{\circ} \mathrm{C}$ to $600^{\circ} \mathrm{C}$. The TG curve showed $4 \%$ water loss and $15 \%$ template loss for the as-synthesized MCM-22, while $10 \%$ water loss was observed in modified HMCM-22. The result is shown in Figure S5 of Supplementary Information (SI).

The $\mathrm{N}_{2}$ adsorption/desorption analysis revealed zeolite's textural properties like surface area and pore size as described in Table 1 . The formation of mesoporosity in modified MCM-22 was supported by its high surface area. Modified MCM-22 shows high surface area and mesoporosity with a major difference in the external surface area. Moreover, parent MCM-22 synthesized using two different silica sources showed the difference in surface area and porosity distributions with each other indicating influence of different silica sources during synthesis.

The influence on silica and alumina amount after modifications were analysed by energy dispersive Xray analysis. There was no significant change in silica/alumina ratio of swelling-sonicated modified MCM22, while alkali-treated modified MCM-22 shows desil- 
ication due to high $\mathrm{pH}$ of gel, resulting in a decrease in the silica/alumina ratio, about half of that of parent MCM-22.

Layered zeolite MCM-22 has acidic nature, which has great applications in acid catalysed organic reactions. ${ }^{19}$ The acidic nature of the catalyst was observed by TPD of ammonia (Figure 3). The TPD pattern confirms the presence of acid sites and strength of the acid sites. Deconvolution of ammonia shows acid sites as weak, medium and strong sites according to the temperature of the release of ammonia from these sites over a large temperature range. The first peak $\left(<200^{\circ} \mathrm{C}\right)$ represented mainly physisorbed ammonia molecules. The second peak $\left(200^{\circ} \mathrm{C}-400^{\circ} \mathrm{C}\right)$ was associated with ammonia molecules adsorbed on hydroxyl groups. The third peak $\left(>400^{\circ} \mathrm{C}\right)$ was associated with dehydroxylation and strong acid sites. ${ }^{20}$ The first peak at around $183^{\circ} \mathrm{C}$ for parent and modified samples are assigned to the weak acid sites and physically adsorbed ammonia. Modified MCM-22 seems to have higher acidity showing a peak with higher peak area at around $550^{\circ} \mathrm{C}$. On the other hand, the alkali-treated MCM-22, shows lesser acidity having insignificant acid sites compared to the parent sample. The amount of ammonia desorbed from zeolitic acid sites is shown in Table 2.

\section{Conclusions}

Synthesis of MCM-22 with different silica sources yielded microporous materials with different crystallinity, while modified MCM-22(P) showed partial mesoporous properties. The comparative study of parent MCM-22 and modified MCM-22 revealed significant changes in physicochemical properties. The silica/alumina ratio also changes after modification indicating the influence of swelling-sonication and alkalitreatment method as a novel development of catalyst. Surface area and pore size analysis after modification suggest presence of mesoporosity having high surface area. These synthesized and modified materials would be further studied in various organic reactions to complement their physicochemical characteristics. Processing of such layered zeolites could be extended to broad application in petrochemical and fine chemical industries.

\section{Supplementary Information (SI)}

The XRD plot of as-made and calcined MCM-22 prepared by two different silica sources are reported as Figures S1 and S2 (Supplementary Information). FE-SEM, FTIR and TG analysis of MCM-22 and modified MCM-22 are reported as
Figures S3-S5 (Supplementary Information) and are available at www.ias.ac.in/chemsci.

\section{Acknowledgements}

Council of Scientific and Industrial Research (CSIR) National Chemical Laboratory (NCL), Pune, provided great support to do instrumental analysis and explore our ideas for research applications. Riddhi Thakkar acknowledges DST, India, for a research fellowship (Inspire fellowship). The authors would also like to thank Dr. Satyanarayana Chilukuri and his research team for helpful technical discussions.

\section{References}

1. Rubin M K and Chu P 1990 Composition of synthetic porous crystalline material, its synthesis and use U.S. Patent 4,954,325

2. Guray I, Warzywoda J, Bac N and Sacco Jr A 1999 Synthesis of zeolite MCM-22 under rotating and static conditions Micropor. Mesopor. Mat. 31241

3. Chlubna P, Roth W J, Zukal A, Kubu M and Pavlatova J 2012 Pillared MWW zeolites MCM-36 prepared by swelling MCM-22P in concentrated surfactant solutions Catal. Today 17935

4. Roth W J, Nachtigall P, Morris R E and Čejka J 2014 Two-dimensional zeolites: Current status and perspectives Chem. Rev. 1144807

5. Leite R C N, Sousa B V and Rodrigues M G F 2009 Static synthesis and characterization of MCM-22 zeolite applied as additive in fluid catalytic cracking operations Braz. J. Pet. Gas. 075-082 1982-0593

6. Wieslaw J Roth, Nachtigall P, Morris R E and Čejka J 2014 Two-dimensional zeolites: Current status and perspectives Chem. Rev. 1144807

7. Zhicheng L, Shaodian S, Bozhi T, Jinyu S, Bo T and Dongyuan Z 2004 Static synthesis of high-quality MCM22 zeolite with high $\mathrm{SiO}_{2} / \mathrm{Al}_{2} \mathrm{O}_{3}$ ratio Chin. Sci. Bull. 49556

8. Ji Y-J, Xu H, Wang D-R, Xu Le, Ji P, Wu H and Wu P 2013 Mesoporus MCM-22 zeolites prepared through organic amine-assisted reversible structural change and protective desilication for catalysis of bulky molecules ACS Catal. 31892

9. Corma A, Fornes V, Pergher S B, Maesen Th L M and Buglass J G 1998 Delaminated zeolite precursors as selective acidic catalysts Lett. Nat. pp. 353-356, 396

10. Marques A L S, Monteiro J L F and Pastore H O 1999 Static crystallization of zeolites MCM-22 and MCM-49 Micropor. Mesopor. Mat. 32131

11. Diaz U and Corma A 2014 Layered zeolitic materials: An approach to designing versatile functional solids Dalton Trans. 4310292

12. Moliner M, Willhammar T, Wan W, Gonzalez J, Rey F, Jorda J, Zou X and Corma A 2012 Synthesis design and structure of a multipore zeolite with interconnected 12and 10-MR Channels J. Am. Chem. Soc. 1346473

13. Hernandez-Rodriguez M, Jorda J L, Rey F and Corma A 2012 Synthesis and structure determination of a new microporous zeolite with large cavities connected by small pores J. Am. Chem. Soc. 13413232 
14. Leonowicz M E, Lawton J A, Lawton S L and Rubin M K 1994 MCM-22: A molecular sieve with two independent multidimensional channel systems Science $\mathbf{2 6 4}$ 1910

15. Bellussi G, Carati A, Rizzo $\mathrm{C}$ and Millini $\mathrm{R}$ 2013 New trends in the synthesis of crystalline microporous materials Catal. Sci. Technol. 3 833

16. Octaviani S, Krisnandi Y K, Abdullah I and Sihombing R 2012 the effect of alkaline treatment to the structure of ZSM5 zeolites Makara J. Sci. 16 155

17. Miltenburg van A, Menorval de L C and Stocker M 2011 Characterization of the pore architecture created by alkaline treatment of HMCM-22 using ${ }^{129} \mathrm{Xe}$ NMR spectroscopy Catal. Today $\mathbf{1 6 8}$ 57
18. Xiao W, Wang F and Xiao G 2015 Performance of hierarchical HZSM-5 zeolites prepared by $\mathrm{NaOH}$ treatments in the aromatization of glycerol $R S C A d v .1$

19. Venuto P B 1994 Organic catalysis over zeolites: A perspective on reaction paths within micropores Microporous Mater. 2297

20. Lok B M, Marcus B K and Angell C L 1986 Characterization of zeolite acity. II. Measurement of zeolite acity by ammoinia temperature programmed desorption and FTIR. spectroscopy techniques Zeolites 6185

21. Szostak R 1998 In Molecular Sieves: Principles of Synthesis and Identification $2^{\text {nd }}$ edn. (London: Blackie Academic and Professional)

22. Bandyopadhyay R 1997 Catalytic Alkylation and Transalkylation Reactions over Large Pore Zeolites Beta, $Y$ and ZSM-12 (CSIR-NCL, Pune University, Pune, India) 